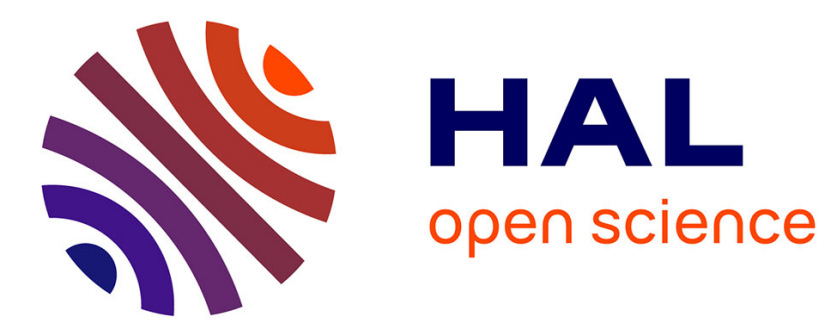

\title{
Marianne Itxer, 8 ans et déjà mariée
}

Christophe Juhel

\section{To cite this version:}

Christophe Juhel. Marianne Itxer, 8 ans et déjà mariée : Chronique juridique et judiciaire d'un mariage précoce célébré en Roussillon au XVIIe siècle. Christophe Juhel. Rôles, statuts et représentations des femmes en Roussillon et en Europe méridionale du Moyen-âge au XIXe siècle, Presses Universitaires de Perpignan, pp.53-64, 2017, 10.4000/books.pupvd.4715 . hal-02462313

\section{HAL Id: hal-02462313 https://hal-univ-perp.archives-ouvertes.fr/hal-02462313}

Submitted on 4 Feb 2020

HAL is a multi-disciplinary open access archive for the deposit and dissemination of scientific research documents, whether they are published or not. The documents may come from teaching and research institutions in France or abroad, or from public or private research centers.
L'archive ouverte pluridisciplinaire HAL, est destinée au dépôt et à la diffusion de documents scientifiques de niveau recherche, publiés ou non, émanant des établissements d'enseignement et de recherche français ou étrangers, des laboratoires publics ou privés. 


\title{
MARIANNA ITXER, 8 ANS ET DÉJÀ MARIÉE : CHRONIQUE JURIDIQUE ET JUDICIAIRE D'UN MARIAGE PRÉCOCE CÉLÉBRÉ EN ROUSSILLON AU XVII SIÈCLE
}

\author{
Christophe JUHEL
}

Sous l'Ancien Régime en France l'âge du mariage est plutôt tardif. Avec de fortes variations entre les régions, les milieux sociaux, les villes et les campagnes, il ne cesse de s'élever entre les XVI ${ }^{\mathrm{e}}$ et XVIII ${ }^{\mathrm{e}}$ siècles. À la veille de la Révolution il est en moyenne de 27-28 ans pour les hommes et 24-25 ans pour les femmes ${ }^{1}$. Cela explique peut-être le désintérêt des jurisconsultes des dernières décennies de l'Ancien Régime pour la question des mariages précoces. À peine y consacrent-ils quelques lignes dans leurs traités ou recueils de jurisprudence ${ }^{2}$ pour répéter les mêmes principes. Tous s'accordent aisément sur le fait que le mariage est possible entre un garçon et une fille ayant tous deux atteint l'âge de la pubertés. Un âge "que les loix ont fixé à quatorze ans complets pour les masles et douze ans pour les filles $»^{4}$. Avant cet âge nul "ne peut prétendre au sacrement de mariage $»^{5}$. Mais si les impubères ne peuvent se marier, ils sont en revanche aptes à se fiancer.

1 - S. Beauvalet-Boutouyrie, La démographie à l'époque moderne, Belin, 1999, 334 p., p. 118; S. MinVielle, La famille en France à l'époque moderne (XV|e-XVIlle siècle), Armand Colin, coll. U - Histoire, 2010, 299 p., p. 47; D. GodineAU, Les femmes dans la France moderne (XVle-XVIIle siècle), Armand Colin, coll. U - Histoire, 2015, 306 p., p. 32; F. LeBRUN, La vie conjugale sous I'Ancien Régime, Armand Colin, 1993, 179 p., p. 32.

2 - Parmi ceux qui font exception figurent Pothier et $D^{\prime}$ Espeisses qui consacrent quelques paragraphes à cette problématique : R.-J. POTHIER, Traité du contrat de mariage, t. 1, Paris, 1771, p. 99-101; A. D’EsPeISSES, Les ceuvres de M. Antoine $d^{\prime}$ Espeisses, Lyon, 1685, t. 1, p. 149-150.

3 - C.-J. FerRIËRE, Traité de droit et de pratique, Paris, 1755, t. 2, p. 277.

4 - C. Horry, Traité du mariage chrétien fait selon les loix de l'Église et les maximes du royaume, Paris, 1695, p. 2. L'âge de la puberté a été numérisé en droit romain pour éviter l'examen physique. De même, pour Argou, le « bas âge » est le premier des empêchements. «II faut que ceux qui se marient, soient en état d'avoir des enfans; ce qu'on a déterminé, suivant le droit romain, aux mâles à quatorze ans, et aux filles à douze ». Il ajoute que « la vieillesse n'est point un empêchement ». Argou, Institution au droit françois, t. 2, Paris, 1764, p. 7.

5 - C. HORRY, op. cit., p. 2. 
Selon le Traité du sacrement de mariage de l'abbé Alabert «les enfants qui n’ont pas atteint l'âge de puberté peuvent validement se promettre, mais quand ils ont atteint quatorze ans il dépend d'eux de retirer leur parole $»^{6}$. Une promesse de mariage ou des fiançailles consenties par un impubère peuvent donc être rompues par lui une fois qu'il est pubère.

Ainsi, seul le mariage de mineurs pubères est possible à condition que le ministre du culte le célébrant ait reçu « le consentement des pères et mères, tuteurs et curateurs " des intéressés ${ }^{7}$. Une règle que confirme la législation royale, notamment l'article 40 de l'ordonnance de Blois de mai 1579 aux termes de laquelle il est enjoint aux "curez, vicaires ou autres de s'enquérir soigneusement de la qualité de ceux qui voudront se marier; et s'ils sont enfans de famille, ou estans en la puissance d'autrui [il leur est défendu] étroitement de passer outre à la célébration desdits mariages, s'il ne leur apparoit du consentement des pères, mères, tuteurs ou curateurs, sur peines d'estre punis comme fauteurs du crime de rapt ${ }^{8}$. L'article $1^{\text {er }}$ de l'ordonnance de $1639^{9}$ et l'article 2 de l'édit de mars 1697 réitèrent cette disposition destinée à assurer la maîtrise des unions aux familles ${ }^{10}$.

Pour autant, en déduire que les mariages d'impubères auraient disparu en France serait bien hâtif. Jean Noguer, juge au Conseil souverain de Roussillon et professeur à l'Université de Perpignan, relève dans son Traité des crimes $^{11}$ un cas soumis à la plus haute juridiction de la province à la fin du XVII siècle, celui de Marianna Itxer, native d'Elne, mariée en 1687 par sa mère avant même d'avoir célébré son neuvième anniversaire. Un cas d'espèce dont l'étude est très révélatrice de la place que peut occuper bien malgré elle une jeune fille au sein de la famille (I) et des conséquences judiciaires pouvant en découler (II).

\section{Une petite fille devenue un enjeu patrimonial}

Le cadre familial au sein duquel Marianna Itxer a grandi est plutôt banal. Il s'est construit au gré d'événements heureux, tels que des mariages et naissances, et malheureux que sont les décès (A). Autant d'étapes que retracent les actes juridiques qui les ont parfois accompagnées et qui ont conduit à terme à ce mariage précoce et précipité (B).

6 - P. Alabert, Traité du sacrement de mariage avec les plus belles décisions prises du droit canonique et civil, Toulouse, 1699, p. 8.

7 - C.-J. FERRIËRE, Nouvelle introduction à la pratique ou Dictionnaire des termes de pratique de droit, d'ordonnances et de coutumes avec les juridictions de France, t. 2, Paris, 1734, p. 253.

8 - A. Jourdan, F.-A. IsAmBerT et DeCRUSy, Recueil général des anciennes lois françaises, Paris, 1827, t. 14, p. $391-392$.

9 - II impose également au curé de recevoir « le consentement des pères, mères, tuteurs ou curateurs síls sont enfans de famille ou en la puissance d'autrui ». A. JouRdan, F.-A. ISAMBERT et DECRUSY, Recueil... t. 16, p. 521.

10 - A. Jourdan, F.-A. IsAmbert et DeCRUSY, Recueil... t. 20, p. 289.

11 - Arch. Dép. des Pyrénées-Orientales, MSS 21 (toutes les références ci-après sont tirées de ce fonds d'archives). 


\section{A. Un décor familial banal}

Marianna Itxer est la fille de Pere Itxer, boucher à Elne, et d'Anna Delbre ${ }^{12}$, tous deux veufs ${ }^{13}$ au moment de leur union ${ }^{14}$. Pere Itxer, veuf depuis un mois à peine ${ }^{15}$, a déjà un fils, Augustí, qui a presque 10 ans $^{16}$. À la naissance de Marianna, l'année suivante, il est désigné pour être son parrain ${ }^{17}$. Trois ans plus tard, naît la petite sœur de Marianna, Maria Theresa, dont le parrain est Miquel Cruzat, un ami de son père ${ }^{18}$ qui jouera un rôle central dans les premières années de sa vie.

Le foyer qui vient de se constituer et d'accueillir successivement deux filles subit ensuite deux décès. Le premier intervient au début du mois d'avril 1683. C'est celui de Pere Itxer qui, sentant sa dernière heure arriver, rédige un testament devant $M^{e}$ Bernat March où il désigne comme héritier son fils Augustí, tout juste âgé de 14 ans, et lui attribue Jaume Mir, pagès de Baho, comme tuteur. Par ce même acte il fait le legs de 100 doublons d'or à chacune de ses filles et nomme pour administrer ces sommes jusqu'à leurs mariages respectifs Miquel Cruzat ${ }^{19}$. Il importe de relever que si Pere Itxer fait un legs à son épouse, il ne la désigne ni pour être tutrice de leurs enfants, ni pour gérer les sommes qu'il alloue à titre de dot à leurs filles. Il semble qu'il soit courant de confier la charge de tuteur à une mère ${ }^{20}$ mais pas à une marâtre; aussi Anna Itxer ne pouvait-elle espérer la recevoir. En revanche, le fait que Pere Itxer ait confié la gestion des dots de ses filles à Miquel Cruzat ne traduit pas une confiance totale de sa part envers sa seconde épouse. Quelques jours après avoir fait consigner ses dernières volontés, il meurt puis est enterré à Elne le 12 avril $1683^{21}$. Quatre ans plus tard, Augustí, frère aîné de Marianna, meurt alors qu'il n'a pas atteint l'âge de 20 ans. Il prend le soin, le 6 octobre 1687, de faire rédiger des codicilles par le notaire d'Elne ayant pris acte des dernières volontés de son père, $\mathrm{M}^{\mathrm{e}}$ March, où il institue Marianna héritière universelle ${ }^{22}$. Il meurt le 7 octobre et est enterré le 8 en présence de Bernat March et de Rafel Cruzat ${ }^{23}$.

12 - Anna Geli, baptisée le 11 octobre 1656 à Elne (CCM 5, fo 163 ro), est la fille de Miquel et Anna Geli habitants d'Elne. Elle a épousé Hieronim Delbre, veuf, le 23 juin 1675 (CCM 4, fo 75 vo. Une première union de courte durée car il a été enterré le 16 décembre 1676 (CCM 4 f० 128 ro).

13 - Sur la question du veuvage sous l'Ancien Régime voir S. BeAUVALET-BOUtOuYRIE, Être veuve sous l'Ancien Régime, Belin, 2001, 415 p. et Les femmes à l'époque moderne (XVle-XVIIle siècles), Belin, 2003, 271 p., p. 17 et sq.

14 - ADPO, CCM 4, f० 78 v․ Mariage célébré le 22 décembre 1677 à l'église d'Elne.

15 - CCM 4, f० $128 v^{\circ}$. Theresa ltxer a été enterrée le 21 novembre 1677.

16 - CCM 5, fo 245 ro. Joseph Augustí Emmanuel Francisco Itxer lorthographié « Hitgé » dans le registre) fils de Pere et Theresa ltxer a été baptisé le 25 mars 1668 à Elne.

17 - CCM 5, fo 235 rº $^{\circ}$ Baptême de Marianna ltxer célébré le 17 novembre 1678.

18 - CCM 4, fo $87 v^{\circ}$. Baptême célébré le 13 février 1681.

19 - 3E1 / 6776. Testament de Pere Itxer du 6 avril 1683; 2B 1296. Legs de Pere Itxer reçu par Bernard March notaire, le 6 avril 1683.

20 - S. Beauvalet-Boutouyrie, Etre veuve... op. cit., p. 272 sqq. En droit romain, il a fallu attendre le Bas Empire, et notamment une constitution des empereurs Valentinien et Théodose le Grand de 390, pour que l'exclusion des femmes de la tutelle de leurs enfants au décès du père subisse ses premières entorses. Voir C. LEFEBVRE, Leçons d'introduction générale à I'Histoire du droit matrimonial français (cours de doctorat), Paris, 1900, p. 239-240.

21 - CCM 4, fo $135 v^{\circ}$. Enterrement de Pere Itxer le 12 avril 1683.

$22-2 B 1296$.

23 - CCM 7, f० $15 v^{\circ}$. Enterrement d'Augustí ltxer le 8 octobre 1687. 


\section{B. Un mariage précoce et précipité}

L'idée de marier au plus vite cette jeune fille, devenue héritière de ses père et frère, n'a probablement pas germé immédiatement dans l'esprit d'Anna Itxer. Il a fallu qu'elle reçoive la convocation, datée du 10 octobre, faite par l'avocat fiscal de la cour de baille d'Elne au conseil des parents, amis et voisins devant se tenir quatre jours plus tard, pour imaginer et mettre en œuvre ce stratagème destiné à conserver la mainmise sur le patrimoine familial.

Au décès de son frère, Marianna a presque 9 ans et se trouve être l'héritière universelle du patrimoine familial qu'il vient de lui léguer. Ce dernier n’a pas établi de tuteur testamentaire, son tuteur étant lui aussi décédé24, Anna Itxer a les mains libres pour quelques jours. En sa qualité de mère de Marianna, elle estime être sa tutrice légitime, ce que est conforme aux usages de l'époque. Elle entend peutêtre aussi éviter la désignation d'un tiers comme tuteur de ses filles. Son mari ayant désigné Miquel Cruzat comme administrateur des legs qu'il a faits à leurs filles, elle ne doit pas imaginer pouvoir être nommée tutrice ${ }^{25}$.

Aussi conclut-elle des pactes de mariage devant Joan Baptista Delaris, notaire d'Elne, le 13 octobre 1687, puis fait célébrer le mariage de sa fille avec un jeune homme, Joseph Calmetes, âgé de 18 ans, présenté comme pagès et garde du duc de Noailles ${ }^{26}$. Le choix d'un autre notaire que celui de la famille est révélateur d'une volonté de ne pas ébruiter l'union avant qu'elle n'ait eu lieu. Ce mariage est finalement célébré dans l'église d'Elne ce jour, à huit heures du soir ${ }^{27}$, par Francès Calmetes, prêtre à Elne, oncle du marié. C'est alors que débute la phase judiciaire de l'affaire.

\section{Une intervention salutaire de la justice}

La justice intervient dans la vie de Marianna Itxer en deux temps puisqu'il faut en premier lieu lui désigner un tuteur (A) pour ensuite invalider le mariage (B) contracté avec Joseph Calmetes.

\footnotetext{
24 - Ce que signale la requête adressée par Anna ltxer du 13 janvier 1688 au Conseil souverain du Roussillon.

25 - Il faut souligner que si Anna ltxer avait été nommée tutrice et que sa fille avait été pubère, le mariage aurait été valide. C.-J. FeRRIËRE, Traité... op. cit., p. 283.

$26-2 B 1296$.

27 - Registre paroissial d'Elne, fo 16: «Als tretza 13 del mes de octobre any mil sy sents vuitenta set 1687, jo Francisco Calmetas curat de Elna, assistit al matrimoni celebrat entre Joseph Calmetas guarda del excellentissim duch de Noallas y entre Marianna Itxer donzella de Elna en la seu de Elna segons lo rito de santa mara la yglesia y lo rito del sagrat concili de tento en presentia y testimonis de Mo Joseph Carrera beneficiat de la seu de Elna, Pera Esteva Oveller de Elna, Joan Depabes brasser de Elna y Pera Esteva menor pastor y no saven de scriurer y o firmo per ells. Francisco Calmetas. Fou celebrat als vuit horas de tarda. Joseph Carrera pre beneficiat de Elna. »
} 


\section{A. Une mise sous tutelle immédiate}

La rapidité avec laquelle le mariage a été célébré tient à ce que le 10 octobre l'avocat fiscal près le tribunal ordinaire d'Elne a convoqué le conseil des parents, amis et voisins des pupilles Itxer pour leur attribuer un tuteur ${ }^{28}$. Cette initiative apparaît classique dans la mesure où ce type de procédure est ouvert lorsqu'un père décède ${ }^{29}$, alors même que la mère et d'autres parents sont en vie $\mathrm{e}^{30}$.

Pour autant, plusieurs indices font présumer que cette situation sort de l'ordinaire. En effet, le conseil des parents, amis et voisins comporte vingt personnes, ce qui est largement au-dessus de la moyenne habituelle. En effet, le quorum

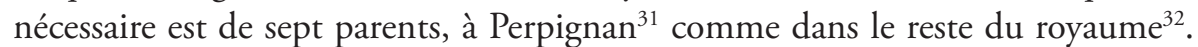
Un arrêt de règlement du Conseil souverain de Roussillon du 20 mars 1728 le confirme $^{33}$. La présence d'autant de parents et de représentants de l'administration villageoise révèle sans doute l'importance de l'enjeu patrimonial et financier de cette affaire ${ }^{34}$. Ensuite, l'empressement d'Anna Itxer à conclure ce mariage laisse à penser qu'elle n'imaginait pas obtenir la charge de tuteur à l'issue de ce conseil. En effet, la tutelle est une " charge virile ", dont les femmes sont naturellement exclues sauf s'il s'agit de la mère ou d'une aïeule des pupilles. Ferrière affirme qu'à défaut de tuteur testamentaire, la mère ou l'aïeule jouit de la tutelle légitime sur les enfants, sans intervention du juge et sans qu'il soit besoin de la nommer ${ }^{35}$.

28 - II existe trois types de tutelle : la tutelle testamentaire réglée par le testament du parent décédé, la tutelle légitime qui est attribuée au plus proche parent des pupilles et la tutelle dative confiée par jugement ou un magistrat.

29 - II semble que ce soit une règle générale (J.-P. BARDET, « Les procès-verbaux de tutelle : une source pour la démographie historique », Mesurer et comprendre. Mélanges offerts à Jacques Dupâquier, PUF, 1993, p. 1-22) et que l'inverse n'arrive jamais. Ainsi « la mort de la mère ne change pas la situation des enfants ». P. OURLIAC et J.-L. GAZZANIGA, Histoire du droit privé français de l'An mil au Code civil, Albin Michel, 1985, p. 277. Selon Guy Brunet le père de famille dispose d'une « tutelle légitime » qui ne nécessite pas l'intervention de la justice. Formulation pouvant prêter à confusion car, juridiquement, la tutelle légitime, qui est dévolue au plus proche parent, implique parfois le concours de la justice. G. BRUNET, "Le juge et l'orphelin. Des assemblées de parents aux conseils de famille, XVIII--XIXe siècles », Annales de démographie historique, 2012/1, n 123, p. 225-247, p. 227. L'expression « tutelle naturelle » utilisée par Anna Lefebvre-Teillard paraît mieux choisie. A. LEFEBVRE-TellLARD, Introduction historique au droit des personnes et de la famille, PUF, 1996, 475 p., p. 422. Pour Scarlett Beauvalet-Boutouyrie le père est un « tuteur naturel ». S. Beauvalet-BoutouyRIE, op. cit., p. 273.

30 - C. PerChE, «La tutelle dative prononcée par les juges du bailliage de Perpignan au XVIIle siècle », G. LARGUIER (dir.), Transmettre et échanger en Roussillon et en Languedoc XVle-XVIIle siècle, PUP, 2011, p. 15-28.

31 - C. PERCHE, op. cit., p. 17-18.

32 - J.-P. BARDET, op. cit.

$33-2 \mathrm{~B} 900$.

34 - La procédure comporte quelques pièces relatives à la contestation par Anna ltxer d'actes et de frais de gestion du patrimoine Itxer. 2B 1296.

35 - J.-A. FERRIERE, Traité des tutelles divisé en quatre parties, Toulouse, 1766, 560 p., p. 21. II infirme la formule de Loisel selon laquelle « Les tutèles sont datives ». A. LoISEL, Institutes coutumières, Paris, 1607, IV, p. 14. Une formule qui, légèrement déformée, se retrouve chez Bourjon et Pothier selon lesquels « toutes tutelles sont datives » ou « les tutelles sont datives, non légales ». Pothier reconnaît cependant qu'il s'agit là de la règle commune en droit coutumier français qui se distingue ici du droit romain où coexistent les tutelles légitime, testamentaire et dative. Bourjon, Le droit commun de la France et... t. l, p. 47.M. DupIN, OEuvres de Pothier, t. 8, Traité des personnes et des choses, Paris, 1825, p. 63. Par ailleurs, de nombreux auteurs affirment au XVIIle siècle qu'en vertu d'un « droit du sang et de la nature », " les père et mère sont les tuteurs nez de leurs enfants ». A. LefebVre-TellLard, op. cit., p. 424-425. 
Il est vrai que généralement «à la mort de son époux, la veuve se voit naturellement placée à la tête du foyer conjugal ${ }^{36}$. Si quatre ans plus tôt Pere Itxer a expressément écarté son épouse par testament au profit de Jaume Mir pour exercer cette tutelle, c'est parce que la mère de ses deux filles n'était que la marâtre du fils de son premier lit. Or, en 1687, ce dernier étant décédé, plus rien ne semblait en apparence s'opposer au retour en grâce d'Anna Itxer. D'autres éléments doivent nous échapper car les progrès des droits de la mère, constatés depuis le Bas Empire en matière de tutelle et tout particulièrement pour l'autorisation au mariage des filles ${ }^{37}$, n'ont pas produit leurs effets dans cette affaire. Le comportement fautif d'Anna Itxer n'explique pas tout. Si, en sa qualité de mère, elle avait été sûre d'être nommée tutrice, pourquoi aurait-elle fait célébrer ce mariage si tôt? Sa personnalité était peut-être en cause : en 1683, à l'âge de 27 ans, elle était veuve pour la seconde fois. Elle apparaissait peut-être comme vénale ou prédatrice, son premier mariage n'ayant duré qu'un an et demi et ayant épousé Pere Itxer un an plus tard alors qu'il était veuf depuis un mois seulement.

Toujours est-il que le 14 octobre, une fois le mariage de Marianna célébré, vingt personnes siègent dans ce conseil des parents, amis et voisins ${ }^{38}$. Neuf sont des parents : il s'agit d'Anna Itxer, mère des pupilles; Francisco Geli, pagès, oncle par leur mère; Domingo Fullà, oncle par alliance; Pau, Antoni et Emmanuel Moliner, cousins germains de leur mère et donc oncles des pupilles; Francisco et Pau Palat, oncles par alliance car mariés à des cousines germaines de leur mère, et Galderich Pus, cousin germain d'Augustí Itxer leur défunt frère. Six sont des membres de l'administration du village : Miquel Cruzat et Agustí Denís, bailles d'Elne; Emmanuel Deulosal, Joan Antoni Regencós et Jaume Pla, consuls et Francisco Vergés, clavaire. Enfin, y participent cinq habitants de la ville présentés comme des amis des pupilles et de leurs parents : le notaire Bernat March, Joseph Fort, Francès Ramon, Joan Roés et Miquel Rives. Tous répondent présents et prêtent le serment de donner un avis «bon et fidèle » sur le contenu de la requête de l'avocat fiscal.

D'emblée, il saute aux yeux que dans ce conseil ne siège qu'une seule femme, Anna Itxer, mère des pupilles. Toutes les autres ont été sciemment écartées ${ }^{39}$, ce qui ressort évidemment de la convocation de parents par alliance, des hommes mariés aux tantes des pupilles mises, elles, de côté. Caroline Perche révèle qu’à Perpignan «si quatre procédures signalent la présence de quelques tantes, grand-

36 - S. BeaUVAlet-BOUtOUYRIE, op. cit., p. 271.

37 - Une constitution de 371 confère successivement au père, puis à la mère et enfin à défaut de ceux-ci aux proches parents le droit de consentir au mariage d'une mineure de 25 ans. C. LeFEBVRE, op. cit., p. 199.

39 - P. PetOT, Histoire du droit privé français. La famille, éd. Loysel, Paris, 1992, p. 509. 
tantes ou tantes à la mode de Bretagne, ces dernières sont les épouses d'autres nominateurs, ne sont pas nécessaires au quorum et leur avis n'est jamais comptabilisé parmi les suffrages. Aucune d'entre elles n'est d'ailleurs nommée tutrice ni même proposée par les membres des assemblées ${ }^{40}$.

De plus, ne siègent que neuf parents ${ }^{41}$, dont Anna Itxer, face aux six membres de l'administration communale ${ }^{42}$ et cinq amis habitants à Elne. Les parents étant moins nombreux que les autres conseillers réunis, ils n'ont donc a priori pas la majorité des voix dans ce conseil de parents, amis et voisins. Il semble ainsi que ce soient les "amis et voisins", pour ne pas dire l'élite ou l'aristocratie locale composée des membres du consulat et de notables du village, qui dominent cette assemblée.

Les avis de chaque membre du conseil sont ensuite successivement consignés. La première à l'exprimer est Anna Itxer qui demande à être tutrice de ses filles. Francisco Geli et Domingo Fullà abondent immédiatement en son sens : une mère se doit d'être éducatrice et protectrice avec ses enfants ${ }^{43}$, quoi de plus naturel que de lui confier juridiquement cette mission. Les autres membres du conseil lui sont bien moins favorables. En effet, Pau, Antoni et Emmanuel Moliner, Galderich Pus, Francisco Palat, Emmanuel Deulosal, Miquel Rives et Joan Roés désignent purement et simplement Miquel Cruzat comme pouvant être tuteur. Pau Palat motive quant à lui son vote par le fait que «ladite Anna Itxer n'a pas procédé comme elle devait ». Le même argument est repris par Bernat March qui ajoute que Pere Itxer avait confiance en Miquel Cruzat. D'autres membres du conseil, font part de cette confiance, tels Francisco Vergés, Joseph Fort, ou encore Joan Antoni Regencós et Francès Ramon qui soulignent que Pere Itxer avait laissé l'administration de la dot de ses deux filles à Cruzat. Les deux bailles ne votent pas pour eux. Miquel Cruzat vote en faveur d'Antoni Moliner, cousin germain des pupilles, alors qu'Agustí Denís dit que Miquel Rives est « capable d'être tuteur». Enfin Jaume Pla vote pour Regencós sans le justifier.

Sur vingt membres du conseil quatorze ont désigné Miquel Cruzat, qui n’a pas voté pour lui-même, tandis qu'Anna Itxer n'a reçu que trois suffrages dont le sien. Bernat March, Miquel Rives, Joan Antoni Regencós et Antoni Moliner ont chacun reçu une voix ${ }^{44}$.

40 - C. PERCHE, op. cit., p. 17-18.

41 - Sur la composition de tels conseils, voir S. MinVIELLE, op. cit., p. 180. Sur la place occupée par les oncles et tantes, voir M. TREVISI, «Oncles et tantes au XVIIIe siècle : au coeur de la parenté, quelle présence, quels rôles? », Histoire, économie et société, 2004, n², p. 283-302.

42 - Les deux bailles, trois consuls et le clavaire de la ville d'Elne.

43 - D. GODINEAU, op. cit., p. 54.

44 - Le décompte des voix fait apparaître vingt-et-un votes pour vingt votants car Joseph Fort a estimé que Miquel Cruzat et Bernat March pouvaient tous deux être tuteurs car Pere ltxer leur faisait confiance. 
Une fois ces avis donnés, Vaquer précise qu'Anna Itxer s'est effectivement rendue " indigne non seulement de la tutelle mais aussi de l'éducation de ses filles " parce qu'elle a marié l'aînée de ses filles qui n'a pas encore neuf ans sans participation de ses autres parents et sans tenir compte de ce qu'un tuteur devait être nommé. Anna Itxer confirme tous les faits et précise, comme il le lui a été demandé, que l'union célébrée à l'église le 13 octobre au soir n'a pas été consommée. Au vu des faits avérés par Anna Itxer et des avis formulés par les membres du conseil, le procureur fiscal requiert que Miquel Cruzat soit nommé tuteur et curateur des pupilles Itxer, ce que Joseph Vaquer, juge de baille d'Elne, décide incontinent le 14 octobre. Le lendemain le juge de baille d'Elne et Agustí Denís décident de placer les deux jeunes filles chez Cécile Cazelles, habitant à Perpignan, afin de les éloigner de leur mère et de la famille Calmetes ${ }^{45}$.

La veuve Itxer ayant fait appel devant le Conseil souverain de Roussillon contre la nomination de Miquel Cruzat comme tuteur de ses filles, qu'elle accuse de dilapider leur patrimoine, cette Cour place les biens des pupilles Itxer sous séquestre et nomme Agustí Denís, le second baille d'Elne, comme gardien et séquestre par arrêt du 30 octobre $1687^{46}$. Miquel Cruzat, plutôt que de subir passivement cette attaque, saisit également les juges suprêmes de la province. Dans l'arrêt qu'ils rendent le 17 décembre 1687, ils se montrent lucides. Ils commencent par rappeler les arguments développés par Miquel Cruzat qui prétend qu'Anna Itxer a agi pour qu'il " n'y ait personne légitime pour poursuivre les procès bien fondez qui sont nécessaires d'introduire et continuer pour faire déclarer la nullité du prettendu mariage que ladite Itxer a voulu faire contracter à Marianna Itxer l'une desdites pupilles agée de huit ans seulement avec le nommé Joseph Calmetes du lieu de St Cebria [...] et d'agir contre ladite Itxer par tous les moyens justes et raisonnables qu'affin lesdites pupilles ne restent sans deffenseur et leurdite mère impunie ". Ils évoquent ensuite les prétentions d'Anna Itxer qui sont purement financières car elle souhaite que les frais de justice causés par l'action de Miquel Cruzat ne soient pas supportés par ses filles. Ils ordonnent finalement à Miquel Cruzat " d'introduire poursuivre et solliciter tous les proces qui seront utilles auxdites pupilles et de fournir aux fraix et dépens qui seront nécessaires, lesquels en raportant les pièces justifficatives luy seront allouez et tenus en compte, et de procéder en tout de conseil d'un habille avocat $»^{47}$.

Au terme de ce premier volet judiciaire de l'affaire Itxer, les deux bailles d'Elne assument la protection des pupilles Itxer : Agustí Denís a reçu la garde de leurs

$\overline{45-2 B} 647$. Ce jugement est mentionné dans l'arrêt rendu par le Conseil souverain le 15 décembre 1687 portant sur les défraiements de Cécile Cazelles. 
MARIANNA ITXER, 8 ANS ET DÉJÀ MARIÉE...

biens et Miquel Cruzat doit diligenter les procès qui leur seront utiles, et notamment poursuivre l'annulation du mariage de Marianna.

\section{B. Une longue procédure d'invalidation du mariage}

La tutelle des pupilles Itxer étant confirmée au profit de Miquel Cruzat, ce dernier saisit l'officialité d'Elne. Les juges d'Église sont en effet compétents pour vérifier qu'aucun empêchement n'existe aux mariages et apprécier ainsi leur validité. L'un des premiers empêchements est constitué par la parenté, deux proches parents ne pouvant se marier. La publication des bans, destinée à faire la publicité du mariage dans la paroisse, est le moyen le plus adapté avec la tenue des registres paroissiaux de baptêmes et mariages, pour savoir si un tel lien existe. Or, en l'occurrence, il n'y a pas eu de publication de bans. Francès Calmetes aurait obtenu une dispense de bans ${ }^{48}$, une "llicentia closa " ${ }^{49}$, afin de célébrer cette union le jour même. La procédure devant l'officialité est initiée au mois d'octobre 1687 mais elle semble n’avoir véritablement débuté qu'en janvier 1688. En effet, l'official décide dès le 31 octobre 1687 d'interroger et examiner la jeune fille mais cet interrogatoire n'a lieu que le 8 janvier 1688. Le 29 janvier 1688 commence une information sur l'absence de publication des bans ${ }^{50}$. L'acte est plutôt sommaire puisqu'il consiste dans la comparution de deux témoins : Pere Rigall, tisserand à lin de Perpignan, et Hiacintó Vaquer, étudiant en médecine, qui tour à tour jurent tous deux connaître ce couple et savoir qu'il n' existe aucun empêchement à ce mariage ${ }^{51}$.

L'official ne s'intéresse plus alors à la validité du mariage qu'au regard de l'empêchement dénoncé par Miquel Cruzat, à savoir le trop jeune âge de Marianna Itxer. Dans la décision qu'il rend très tardivement, le 23 août 1688, il souligne "qu'il conste par l'extrait du batistaire de ladite Itxer qu'elle n'avoit pas atteint l'age puberté et qu'elle en estoit bien esloignée lorsqu'elle a célébré ledit mariage puisqu'elle n’avoit pas encore neuf ans accompli ce qui est une nullité essentielle audit mariage ».

48 - En général les dispenses de bans sont sollicitées pour éviter que l'union ne soit inutilement retardée par de « fausses ou malicieuses dénonciations d'empêchements » émanant de personnes mal intentionnées ou tout autre motif fallacieux. R. Congost, J. Portell, E. Saguer et A. Serramontmany, op. cit. R.-J. Pothier, Traité du contrat de mariage... op. cit., p. 83. \|l s'agit aussi d'éviter les charivaris, voir C. JuHEL, « Indiscipline et désordres de la jeunesse en Roussillon au XVIII siècle », G. Larguier (dir.), Mineurs, minorités; Jeunes, Jeunesse en Roussillon et Languedoc XVI-XVIIle siècle, PUP, 2010, p. 121-144. 49 - En Catalogne l'expression llicències closes, tout comme celle de dispenses de proclames, est équivalente à celle de dispense de bans ou licence pour se marier. Voir l'étude sur le diocèse de Gérone de R. CONGOST, J. PORTELL, E. SAGUER et A. SerRamontmany, "Les dispenses de bans. Une source pour la démographie historique et l'histoire sociale », Populations, 3/2012, vol. 67, p. 549-563.

50 - 2B 1296. Informatio matrimony bannis non publicatis inter Josephum Calmetes loci de Sant Cebria ex una et Mariannam Itxer domicellam ciutatis Elnae partibus ex altera.

51 - 2B 1296: « Petrus Rigall textor lini Perpiniani testis citatus $\S[$ sic] qui juravit $\S$ dicere veritatem $\S$ et interrogatus $\S$ dixit que ell coneix molt be a dits Joseph Calmetes y Marianna ltxer losquals no son parents ni tenan entre si ningun canonich impediment ans be son liberos pera casarse y es veritat per lo jurament que tinch prestat. Hyacinthus Vaquer medicinae scudiosus civitatis Elnae testis atatus que juravit $\S$ dicerre veritatem $\S$ et interrogatus $\S$. Dixit que elle te molt conegut y tractats alsdits Joseph Calmetes y Marianna Itxer losquels son liberos pera casarse y noy ha entre ells ningun canonich impediment y es la veritat per lo jurament que tinch prestat. » 
Il précise ensuite que l'adage malitia supleat atatem ${ }^{52}$ ne peut jouer pour Marianna Itxer pour deux raisons : en premier lieu, "parce qu'il se voit par son audition et ses réponses d'un costé qu'elle n'a aucun dicernement d'esprit touchant le mariage ne scachant à quoy il oblige et pour quoy il se fait, et de l'autre que se pretandeu mariage n'a peu estre consommé » et, en second lieu parce qu'il est apparu aux juges lors d'un examen physique sommaire ${ }^{53}$ de la jeune fille que non est viri potens. En effet, Pothier signale que «si la vigueur [a] devancé l'âge en cette personne et qu'elle [a] donné des preuves de puberté [...] si une jeune fille, mariée avant l'âge de 12 ans, [est] devenue grosse, le mariage [est] valable; car le défaut d'âge ne forme un empêchement de mariage qu'autant qu'il forme une présomption de défaut de puberté; mais dans cette espèce, la présomption est détruite par le fait et la preuve que cette jeune personne a donnée de sa puberté " ${ }^{54}$. Il cite en exemple un arrêt du Parlement de Paris de 1622 rapporté par Jean Bouguier concernant une jeune fille mariée à l'âge de onze ans et veuve quatre mois plus tard, dont l'union fut reconnue licite pour avoir été consommée, la jeune veuve étant tombée enceinte $^{55}$. En effet, il ne faut oublier que l'âge numérisé, fixé à 12 ans, présente l'intérêt de ne pas avoir à vérifier si une jeune fille est nubile, cette épreuve pouvant apparaître comme impudique, mais que la véritable condition matrimoniale est la nubilité. D’Espeisses précise que "selon le droit canon on est présumé estre parvenu à la puberté dès qu’on est capable d'engendrer ». En pareil cas cependant une fille n'échappe pas à «l'inspection faite par les sages-femmes, ez parties propres à génération. [...] Puisque la principale fin du mariage c'est la procréation des enfants [...] il est juste qu'on ne regarde pas au nombre des années de ceux qui se marient, mais seulement à leur capacité d'engendrer $"^{56}$.

En l'occurrence, en l'absence d'indice physique ou "moral " permettant de combattre la présomption d'impuberté de Marianna Itxer l'official déclare « ledit mariage nul et invalide " et interdit " auxdits Calmetes et Itxer de cohabiter ensemble à peyne d'excommunication ». Le vicaire général et official aurait ensuite sanctionné Francès Calmetes, pour avoir indûment célébré cette union ${ }^{57}$.

52 - Littéralement « Malitia supplet aetatem » signifie « La malice supplée à l'âge ». Les juges précisent à ce propos que « l'examen [...] malitia supleat atatem n'est accordé qu'en faveur de ceux qui sont proches de la puberté et que ladite ltxer en estoit esloignée de plus de trois ans ».

53 - 2B 1296. La formule utilisée par les juges est la suivante : « de la disposition du corps de ladite ltxer à la seule conspection de laquelle il paroit que non est viri potens ».

54 - R.-J. PothiER, Traité du contrat de mariage... op. cit., p. 99-100; A. LefEBVRE-TellLARD, Introduction au droit des personnes et de la famille, PUF, coll. Droit fondamental, 1996, p. 106 et 136

55 - J. Bouguier, Arrests de la Cour décisifs de diverses questions tant de droict que de coutumes, Paris, 1647, p. 198 sqq. Bouguier ajoute qu'un arrêt de 1601 rendu en sens opposé a été évoqué lors de ce procès, mais qu'il était fondé sur l'incapacité de la jeune femme à pouvoir prouver la consommation de son union, s'étant remariée quelques mois après le décès de son premier mari.

56 - A. D'ESPEISSES, op. cit., p. 150.

57 - Nous ne disposons pas de cette décision. Elle est mentionnée par les juges du Conseil souverain de Roussillon dans leur sentence du 4 juin 1688 (2B 1296). 
Le 4 juin 1688, avant même que l'officialité ne se soit définitivement prononcée sur la validité du mariage, le Conseil souverain siégeant à Perpignan déboute Anna Itxer de son appel contre la nomination de Cruzat comme tuteur, ordonne le placement des pupilles Itxer dans un couvent et assigne Anna Itxer, Joan Baptista Delaris notaire, Pere Rigall et Hiacintó Vaquer pour être ouïs sur les faits contre eux résultant du procès. Cette affaire aurait pu en rester là mais deux ans plus tard, Miquel Cruzat saisit à nouveau la justice pour protéger les pupilles Itxer. En effet, il demande qu'elles soient déplacées car Anna Itxer et Joseph Calmetes sont parvenus "à gagner quelques personnes " du couvent où elles sont enfermées et leur parlent très souvent. Une requête reçue par le Conseil souverain qui, dans un arrêt du 23 mai 1690, autorise Miquel Cruzat à faire sortir les deux jeunes filles du couvent Notre Dame de Perpignan pour les placer dans un autre couvent de la province ou du Languedoc ${ }^{58}$. Miquel Cruzat choisit alors de les placer dans le couvent des religieuses de Sainte Catherine de Sienne de Perpignan.

C'est finalement en 1691 que cette affaire connaît son épilogue. Le Conseil souverain est saisi par Miquel Cruzat, en sa qualité de tuteur de Marianna, et Marianna elle-même afin d'autoriser cette dernière à " contracter mariage avec Joseph Calmetes du lieu de Sainct Cebria veu qu'elle est déjà en age nubile, que c'est un parti avantageux à ladite pupille et qu'elle le désire pour l'inclination qu'elle a pour ledit Calmetes ". Marianna est à ce moment-là toujours enfermée par ordre de la cour dans le couvent de Sainte Catherine de Sienne. Par arrêt du 11 octobre 1691 la Cour suprême roussillonnaise ordonne qu'avant dire droit sur ces requêtes, une assemblée des parents de Marianna « jusqu'en nombre de huit et à leur défaut des amis ou voisins " doit être convoquée en présence de Vilar, membre de cette cour, pour formuler un avis sur ce mariage ${ }^{59}$.

Ce conseil, tenu le 14 octobre, composé de « quatre parens de ladite pupille deux consuls de ladite cité d'Elne et deux amis et voisins " se déclare favorable à ce mariage parce qu'il est " avantageux à ladite pupille » et que « ledit Joseph Calmetes [est] fort bon garçon de bonnes mœurs et d'honneste famille ». La réduction du nombre de membres du conseil est à remarquer, de même que l'équilibre entre les parents d'une part et les consuls, amis et voisins de l'autre. Certainement que l'issue de la délibération ne faisant pas de doute et n'y ayant aucun empêchement à l'union projetée, il n'était pas utile de réunir davantage de personnes. Aussi le Conseil souverain ne s'oppose pas au mariage et ordonne que Marianna Itxer sorte du couvent pour être " mise entre les mains dudit tuteur pour passer le contract dudit mariage et faire tous les actes à ce nécessaires de son consentement ${ }^{60}$.

$58-2 \mathrm{~B} 1296,2 \mathrm{~B} 866$ fo $^{\circ} 156$ r $\mathrm{r}^{\circ}$ et $2 \mathrm{~B} 650$.

$59-2 B 867$, fo $213 v^{\circ}$ : arrêt du 11 octobre 1691.

$60-2 B 867$, fo $238 v^{\circ}$ : arrêt du 17 octobre 1691 . 
Le mariage est célébré le 28 octobre 1691, non pas à Elne, mais dans la cathédrale St Jean de Perpignan en présence de Miquel Cruzat ${ }^{61}$. Trois ans plus tard, le 5 août 1694, Marianna qui n'a pas encore 16 ans, accouche d'une petite fille prénommée comme elle, dont Francès Calmetes et Anna Itxer sont les parrain et marraine ${ }^{62}$.

Cet épisode de la vie des Roussillonnais sous l'Ancien Régime est instructif à plus d'un titre. Il illustre notamment l'asymétrie des relations hommes-femmes : le conseil de famille est réuni du fait du décès des deux hommes de la famille, l'assemblée des parents, amis et voisins ne comporte aucune femme en dehors de la veuve Itxer et cette dernière n'est jamais nommée tutrice de ses propres filles. Il confirme, si besoin en était, que les affaires familiales sont avant tout d'ordre patrimonial ${ }^{63}$ et intéressent tout autant la famille que les membres de la communauté villageoise, ces derniers étant en nombre égal ou supérieur aux parents dans les conseils des parents, amis et voisins. Un trait qui trahit peut-être la volonté de la communauté d'habitants de préserver le patrimoine de ses familles ou bien d'en tirer profit dès lors que l'occasion s'en présente. La première interprétation attesterait d'une solidarité villageoise tandis que la seconde réduirait ce procès à des luttes d'intérêts où ceux qui sont investis d'une autorité cherchent à profiter financièrement des périodes de faiblesse des familles qui composent leur communauté. Cette affaire est éclairante sur le fonctionnement de la justice roussillonnaise. Elle révèle que la répartition de compétence entre les justices laïque et ecclésiastique est bien établie quant à l'annulation du mariage pour empêchement qui relève de l'official, le juge laïque se cantonnant aux aspects temporels du litige. Elle permet aussi de mesurer le peu de célérité de la justice ecclésiastique puisque l'official a mis trois mois pour interroger Marianna Itxer et six mois supplémentaires ont été nécessaires pour anéantir un mariage dont la nullité ne faisait d'emblée aucun doute. Une extrême lenteur très préjudiciable en matière familiale et qui a conduit le Conseil souverain de Roussillon, comme l'avait rapidement fait le juge de baille d'Elne, à éloigner Marianna Itxer de sa mère pour assurer sa protection, sans attendre l'annulation du mariage par l'officialité d'Elne ${ }^{64}$.

61 - 9NUMI12EDT851-852. Registre paroissial de la cathédrale St Jean, année 169l, fo 102 vo: «Vuy als vint y vuit del mes de octobre del any mil sis cents nonanta hu jo Honorat Ayxart prebere curat de la present iglesia de sant Joan Baptista de la present vila de Perpinya e assistit al matrimoni en fas de santa mara iglesia celebrat entre Joseph Calmetas fill de Nicolau y de Isabel conjuges dell loch de sant Cypria de una part, y Maria Anna Itxe donzella filla de Pere y de Anna conjuges de la ciutat de Elna de part altre. Testimonis Francisco Cruzat batlle de Sant Cypria Claudes Beaufort secretari de Monsieur le president de Prat, Miquel Cruzat y Joan Roés de Elna, los quals se son firmats en fe de que firmo jo. Honorat Ayxart pbre curat, Beaufort, Miquel Cruzat, Joan Roés, Francisco Cruzat ».

62 - 9NUM65CMC8. Registre paroissial d'Elne, année 1694, fo 27 v .

63 - Voir notamment R. NOUGIER, Les relations patrimoniales dans le contrat de mariage : étude sur les techniques patrimoniales en Roussillon (1659-1789), thèse, droit, Perpignan, 2003, 3 t., 810 p.; J. PEYTAVI-DeIXONA, La família nord-catalana: matrimonis i patrimonis: segles XVI-XVIII, Trabucaire, Canet, 1996, $187 \mathrm{p}$.

64 - Cet apparent défaut de synchronisation donne l'impression que les deux procédures sont menées parallèlement et non de concert alors que elles portent sur le même objet et que le dénouement de l'une, pendante devant le Conseil souverain, dépend de l'issue de l'autre, relevant de l'official. 